\title{
Fuzzy Pain Demand Index from an i-Pain System for Assessment of Postoperative Pain via Patient- Controlled Analgesia Using a Multilayer Hierarchical Structure
}

\author{
Jiann-Shing Shieh \\ Department of Mechanical Engineering \\ Yuan Ze University \\ Chung-Li, Taiwan \\ jsshieh@saturn.yzu.edu.tw \\ Chun-Yi Dai \\ Department of Mechanical Engineering \\ National Central University \\ Chung-Li, Taiwan \\ chunyi.dai@gmail.com
}

\author{
Yeong-Ray Wen \\ Department of Anesthesiology \\ Shin Kong Wu Ho-Su Memorial Hospital \\ Taipei, Taiwan \\ yr.wen@yahoo.com.tw \\ Wei-Zen Sun \\ Department of Anesthesiology \\ College of Medicine, National Taiwan University \\ Taipei, Taiwan \\ wzsun@ntu.edu.tw
}

\begin{abstract}
An advanced multilayer hierarchical structure included a personal digital assistant (PDA) device for an $i$-pain system is described in this paper. This PDA is like a messenger that not only records visual analog scales (VASs) and side effects but also collects the patient-controlled analgesia (PCA) data via the RS232 port by wire (i.e., cable) or wireless (i.e., Bluetooth) at time of medical staff visits. In our previous study, a novel fuzzy pain demand (FPD) derived from the interval of each bolus of PCA according to a fuzzy modeling algorithm can show the patients' dynamic demand and past efforts to overcome the postoperative pain. Hence, this study investigated whether FPD index can distinguish the analgesic efficacy using different amounts and combination drugs via six hundred and seventynine patients with upper and lower abdominal, spinal, and extremity procedures.
\end{abstract}

Keywords- $A$ multilayer hierarchical structure; fuzzy pain demand; patient-controlled analgesia; fuzzy modelling

\section{INTRODUCTION}

Although the subjective feeling of pain is extremely hard to quantify, the need for a reliable and valid tool in pain measurement is essential for any clinical practice or trial associated with pain treatment [1]. In most situations, the most commonly used measures, such as visual analog scales (VASs), numerical rating scales (NRSs), and verbal rating scales (VRSs) are sufficiently sensitive to detect the subjective improvement in pain intensity across populations and settings [2]. However, as with all subjective symptoms, an inherent disadvantage in pain rating is its dependence on the patient's verbal reports. The recorded value merely represents a patient's subjective interpretation of the pain experience and the patient's cognitive assignment along the scaling system. This lack of sensitivity in many situations could further limit its clinical implications [3]. In the search for a useful adjuvant to self-reported pain intensity, the patient-controlled analgesia (PCA) device is an important means to develop a reliable, objective, continuous, and on-line index. In conventional PCA systems, consenting patients are provided with a hand-held pushbutton and are instructed to trigger the button when they require pain relief. In our previous research [4], a novel fuzzy pain demand (FPD) index derived from the interval of each bolus of patientcontrolled analgesia (PCA) is designed and documented in a large scale clinical survey. The FPD index is modeled according to a fuzzy modeling algorithm to interpret the selftitration of the drug delivery. It has been found the FPD index modeled from a fuzzy modeling algorithm to interpret the selftitration of the drug delivery can show the patients' dynamic demand and past efforts to overcome the postoperative pain. Moreover, this index could become on-line system to monitor patients' demand or intent to treat their pain so these factors could be entered into a patient's chart along with temperature, blood pressure, pulse and respiration rates when medical practitioners check the patients. Hence, in the present study, we investigated whether FPD index can distinguish the analgesic efficacy using different amounts and combination drugs.

\section{A MULTILAYER HIERARCHICAL STRUCTURE OF THE I- PAIN SYSTEM}

An advanced multilayer hierarchical structure of $i$-pain (where $i$ means information, intelligence, interaction, and internet) system for data collection and interpretation includes the four layers of patients, measurement, web-based and interpreting, as shown in Fig. 1.

\section{A. Layerl 1: Patients (i.e., Patient level)}

This study was approved by the Shin Kong Wu Ho-Su Memorial Hospital Ethics Committee. Six hundred and

Permission to make digital or hard copies of all or part of this work for personal or classroom use is granted without fee provided that copies are not made or distributed for profit or commercial advantage and that copies bear this notice and the full citation on the first page. To copy otherwise, to republish, to post on servers or to redistribute to lists, requires prior specific permission and/or a fee. 


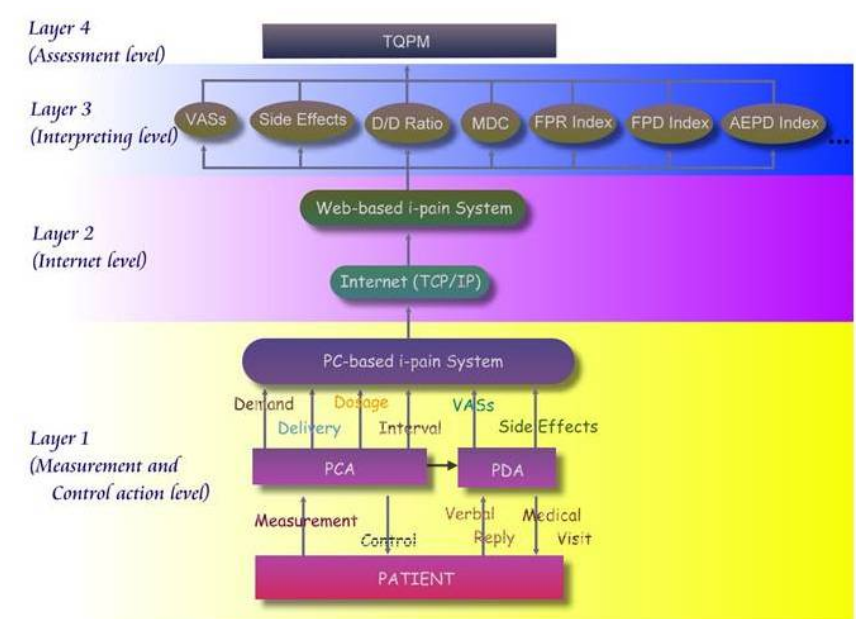

Fig. 1 An advanced multilayer hierarchical structure of $i$-pain system

seventy-nine patients with American Society of Anesthesiologist physical status 1, 2 or 3 for upper and lower abdominal, spinal, and extremity procedures were screened from the $i$-pain database and entered into this study. In order to test our FPD index in this paper, they were tested into four groups between morphine $(1 \mathrm{mg} / \mathrm{ml})$ alone and a combination of morphine $(1 \mathrm{mg} / \mathrm{ml})$ and ketorolac $(1.2 \mathrm{mg} / \mathrm{ml})$ for different dosages. The four groups were: (1) morphine alone with a 1-ml per delivery (MOR-1); (2) morphine alone with a 2-ml per delivery (MOR-2); (3) combination of morphine and ketorolac with a 1-ml per delivery (MOR-K-1); and (4) combination of morphine and ketorolac with a 2-ml per delivery (MOR-K-2). Patients were excluded from the study if they were morbidly obese, unable to understand the use of the PCA or had a history of allergy to either morphine alone or a combination of morphine and ketorolac. According to routine clinical practice, patients were instructed on the correct use of the PCA pump and given standardized PCA education by a PCA team nurse.

\section{B. Layer 2: Measuring patients' demand, inputting} patients' basic information and medical visits via a personal digital assistant (PDA) device (i.e., Measurement level)

PCA has become an established procedure for clinical pain relief. A number of studies have shown the advantages of PCA over regularly scheduled and as-required administration of analgesics. The PCA machine provides a system where the patient operates a hand-held button interfaced to a microprocessor that drives an infusion pump delivering intravenous analgesic. Furthermore, the pain demand and delivery of patients stored inside the PCA device may represent different degrees of pain relief. Hence, the second level is a measuring level that involves an instrument device (i.e., Abbott AIM Plus pump), which collects all the patient demands and delivers a bolus to the patient when they require pain relief. The collected information is in two modules, which provide basic information input and items, and data retrieving from the PCA machine. A basic module provides the information inputs and items, such as the patient height, weight and age, doctor's and nurse's name, drug's name, dosage and concentration, ...., etc. The data retrieving module from the PCA machine provides the number of demands and delivery, the bolus volume, the continuous infusion volume, and the total drug consumption for each day. Another instrument is using popular PDA device for collecting clinical observations. This PDA is like a messenger that not only records visual analog scales (VASs) and side effects but also collects the PCA data via the RS232 port by wire (i.e., cable) or wireless (i.e., Bluetooth) at time of medical staff visits as shown in Fig. 1. Then, the patients' basic information and post-operative records of the PCA and PDA devices are transmitted to a personal computer (PC) to create a comprehensive file in the PC-based i-pain system.

\section{Layer 3: Constructing a web-based i-pain system to collect large scale clinical data (i.e., Web-based level)}

With the large scale clinical data input from PCA and PDA devices via a RS232 communication port, we constructed a comprehensive web-based $i$-pain platform to encompass the high-throughput data acquisition. Hence, level 3 is a webbased level that involves all data files, which are merged offline and uploaded to a web-server PC using standard TCP/IP. The web-based $i$-pain system acts as a database for multimodal electronic patient record information and storing for conventional data analysis and further intelligent analysis.

\section{Layer 4: Data mining of the pain database (i.e., Interpreting level)}

In order to encompass the high-throughput data analysis to yield evidence-based medical information, the data mining of the database is like an interpreting level that involves interpreting patients' pain demand to obtain a delivery pattern, and then interpreting the pain pattern to several indexes (such as fuzzy pain relief (FPR) index, fuzzy pain demand (FPD) index [4], and auditory evoked pain demand (AEPD) index [5]. In addition, the conventional patients' VASs, side effects, mean drug consumption (MDC) and demand/delivery (D/D) ratio are also calculated and measured. In order to show the change of all these values, our clinical analysis data are divided into six periods after the start of PCA: period $1(0-4 \mathrm{~h})$, period $2(4-8 \mathrm{~h})$, period $3(8-12 \mathrm{~h})$, period $4(12-16 \mathrm{~h})$, period $5(16-20 \mathrm{~h})$, and period $6(20-24 \mathrm{~h})$. In this paper, we focus on the test of FPD index via high-throughput data input. In order to understand in more detail how to use patients' pain demand to obtain a delivery pattern, and then interpreting the pain pattern to FPD index, please refer to the description of our previous paper [4].

\section{RESULTS}

Table I is the demographic details of the 4 groups. Table II is the data of pain pattern of delivery (i.e., big pain (BP), small pain (SP) and zero pain (ZP)), FPD index, MDC, D/D ratio, and VASs score at most pain and rest pain at different dosages during 6 intervals. From Table II, the trend of FPD index is similar to MDC and D/D ratio because it was reduced sharply during the first 3 intervals then it was gradually increased at the next 3 intervals regardless of the dosages (i.e., 1 or $2 \mathrm{ml}$ per delivery) and drug types (i.e., MOR or MOR-Keto). However, the FPD index demonstrated a better sensitivity (i.e., $P<0.05$ for 3 intervals) than $\mathrm{D} / \mathrm{D}$ ratio (i.e., $P>0.05$ for all intervals) in 
the morphine and ketorolac combination group than morphine alone, regardless of the dosage ( 1 versus $2 \mathrm{ml}$ per delivery). Moreover, it also demonstrated a better sensitivity (i.e., $P<$ 0.05 for 3 intervals) than MDC (i.e., $P<0.05$ for 2 intervals) at $1 \mathrm{ml}$ per delivery but similar results (i.e., $P<0.05$ for 3 intervals) at $2 \mathrm{ml}$ per delivery as shown in Table III.

However, when FPD index is compared with VAS score in terms of most pain and rest pain, there is no any relationship between these two parameters' pattern at these 6 intervals. As mentioned before, the trend of the FPD index was reduced sharply during the first 3 intervals then it was gradually increased at the next 3 intervals. However, the trend of the VAS index was rather randomized if compared with each interval. But, it may be seen at these 6 intervals that the VAS values for most pain and rest pain were controlled under scores 5 and 3, respectively. The basic concept of the PCA design is to reduce patients' pain to a minimum (i.e., VAS $<3$ for rest pain) and to maintain them at pain-free status in the postoperative period regardless of their efforts. That is why a significant improvement in satisfaction score was seen after the introduction of an acute pain service [6]. Moreover, the Pearson product-moment correlation coefficient has been calculated between FPD and VAS score at most pain and rest pain under four groups (i.e., MOR-1, MOR-2, MOR-K-1, and MOR-K-2) as shown in Table IV. This result indicates that FPD index can only assess the intensity of pain better than D/D ratio and MDC but it cannot replace the VAS which is not entirely determined by the nociceptive stimuli but rather as a result of both sensory-discriminative and emotional-cognitive components of patient's suffering.

\section{DISCUSSION}

In this paper, a PDA device acted as the data collection platform for recording the VASs and side effects when medical doctors or nurses interviewed patients has been built into a multilayer hierarchical structure of $i$-pain system to collect the patients' daily medical information into major server. This PDA is like a messenger that not only records VASs scores and side effects but also collects the PCA data via the RS232 port by wire (i.e., cable) or wireless (i.e., Bluetooth) at time of medical staff visits. All data files are merged off-line and uploaded to a web-server PC using standard web-based TCP/IP. Then, further data is mined by a web-server using intelligent analysis can be obtained in order to determine the relationship between PDA (i.e., VAS and side effects) and PCA (i.e., FPD index, MDC, and $\mathrm{D} / \mathrm{D}$ ratio) data. Furthermore, this PDA device could become real time system to monitor patients' demand or intent to treat their pain when this intelligent data mining approach has been proved to be available in clinical test. Also, if the hospital environment is ready for Wi-Fi wireless technology transmission, the PDA device is able to upload and download to our server database easily inside the hospital so that the pervasive computing at this $i$-pain system will be available. Then, the total quality pain management could be applied into acute pain service and this pain index could be entered into a patient's chart along with temperature, blood pressure, pulse and respiration rates when medical practitioners check the patients.

At present, a total of 8 medical centers in Taiwan have joined to share this $i$-pain system. Cumulative evidence from our preliminary results has yielded fruitful implication that in turn can provide immediate feedback for daily practice [4]. With the large scale of clinical data input, we have successfully constructed a comprehensive platform to encompass the highthroughput data acquisition and systemic analysis to yield a series of evidence-based medical evaluations for modern acute pain service.

\section{ACKNOWLEDGMENT}

The authors wish to thank Taiwan branch of the Hospira Pte. Ltd. for supporting this research.

\section{REFERENCES}

[1] J.T. Farrar, J.A. Berlin, and B.L. Strom, "Clinically important changes in acute pain outcome measures: a validation study," Journal of Pain and Symptom Management, vol. 25(5), pp. 406-411, 2003.

[2] M. Briggs, and J.S. Closs, "A descriptive study of the use of visual analogue scales and verbal rating scales for the assessment of postoperative pain in orthopedic patients," Journal of pain and Symptom Management vol. 18(6), pp. 438-446, 1999.

[3] M.P. Jensen, L. Miller, and Fisher, "Assessment of pain during medical procedures: a comparison of three scales," Clin J Pain, vol. 14, pp. 343349,1998

[4] J.S. Shieh, C.Y. Dai, Y. R. Wen, and W.Z. Sun, "A novel fuzzy pain demand index derived from patient-controlled analgesia for postoperative pain," IEEE Transactions on Biomedical Engineering; 2007 (Accepted).

[5] J.S. Shieh, L.Y. Chen, Y.R. Wen, J.Y. Chen, and W.Z. Sun, "Discrimination of pain intensity level and side effects of postoperative pain using parameters extracted from evoked pain pattern," 3rd International Conference MEDSIP 2006 on Advances in Medical, Signal and Information Processing, Glasgow, Scotland, p. 12, July 2006

[6] M.U. Werner, L. Soholm, P. Rotboll-Nielsen, and H. Kehlet, "Does an acute pain service improve postoperative outcome?" Anesth. Analg., vol. 95, pp. 1361-1372, 2002.

Table I Demographic details of the 4 groups

\begin{tabular}{|c|c|c|c|c|c|c|c|c|}
\hline & Patient & Male & Female & Age & $\begin{array}{c}\text { Lockout Time } \\
(\mathrm{min})\end{array}$ & Height (cm) & Weight (kg) & $\begin{array}{c}\text { PCA Duration } \\
\text { (Day) }\end{array}$ \\
\hline MOR-1 & 255 & 109 & 146 & $56.72 \pm 15.37$ & $7.95 \pm 1.98$ & $154.84 \pm 12.81$ & $58.68 \pm 9.89$ & $5.16 \pm 2.52$ \\
\hline MOR-2 & 226 & 101 & 125 & $54.13 \pm 17.46$ & $9.5 \pm 1.54$ & $159.13 \pm 10.17$ & $70.23 \pm 8.62$ & $4.92 \pm 2.47$ \\
\hline MOR-K-1 & 123 & 47 & 76 & $56.41 \pm 14.59$ & $6.56 \pm 1.52$ & $161.53 \pm 7.87$ & $61.11 \pm 12.91$ & $5.59 \pm 2.34$ \\
\hline MOR-K-2 & 75 & 23 & 52 & $43.55 \pm 12.61$ & $8.7 \pm 1.31$ & $156.96 \pm 11.5$ & $63.54 \pm 14.43$ & $4.13 \pm 2.44$ \\
\hline
\end{tabular}


Table II Data for pain pattern of delivery (i.e., big pain (BP), small pain (SP) and zero pain (ZP)), FPD index, MDC, D/D ratio and VAS score for most pain and rest pain (Data are presented as median $\left(25^{\text {th }}-75^{\text {th }}\right.$ percentiles) for pain pattern, FPD, MDC and $\mathrm{D} / \mathrm{D}$ ratio, and means (SD) for VAS score in Table)

\begin{tabular}{|c|c|c|c|c|c|c|c|c|c|c|c|c|c|}
\hline Group & Time Interval & \multicolumn{2}{|r|}{$0 \sim 4$} & \multicolumn{2}{|r|}{$4 \sim 8$} & \multicolumn{2}{|r|}{$8 \sim 12$} & \multicolumn{2}{|r|}{$12 \sim 16$} & \multicolumn{2}{|r|}{$16 \sim 20$} & \multicolumn{2}{|r|}{$20 \sim 24$} \\
\hline \multirow{8}{*}{$\begin{array}{c}\text { MOR-1 } \\
\text { [255] }\end{array}$} & $\mathrm{BP}(\%)$ & 10 & $(2.5-25)$ & 3 & $(1-8.33)$ & 2 & $(0.73-5)$ & 2 & $(0.73-7.5)$ & 3.1 & $(1-8.33)$ & 2.5 & $(0.83-7.5)$ \\
\hline & $\mathrm{ZP}(\%)$ & 29.17 & $7(15.83-40.83)$ & 20.83 & $3(2.5-35)$ & 16.67 & $(4.17-30)$ & 20 & $(3.32-36.25)$ & 20 & $(3.32-33.33)$ & 12.5 & $(2.33-30)$ \\
\hline & $\mathrm{SP}(\%)$ & 54.17 & $7(31.25-75)$ & 72.5 & $(50-97.5)$ & 80 & $(60-92.08)$ & 75 & $(54.58-100)$ & 79.17 & $7(54.17-100)$ & 82.5 & $(62.5-100)$ \\
\hline & FPD Index & 6.23 & $(4.23-8.47)$ & 3.97 & $(2.84-5.81)$ & 2.21 & $(1.99-5.59)$ & 2.4 & $(1.43-5.13)$ & 2.63 & $(1.43-5.99)$ & 4.2 & $(2.25-5.15)$ \\
\hline & $\mathrm{MDC}(\mathrm{ml} / \mathrm{h})$ & & $(2-5.25)$ & 1.5 & $(0.5-3)$ & 1 & $(0.5-2.1)$ & 1.5 & $(0.5-3)$ & 1.5 & $(0.5-2.5)$ & 1 & $(0.5-2)$ \\
\hline & D/D Ratio & 1.08 & $(1.03-1.35)$ & 1 & $(1-1.05)$ & 1 & $(1-1.04)$ & 1 & $(1-1.05)$ & 1 & $(1-1.06)$ & 1 & $(1-1.05)$ \\
\hline & VAS (Most Pain) & & $2.05 \pm 2.93$ & & $3.56 \pm 2.63$ & & $2.63 \pm 3.50$ & & $2.86 \pm 2.73$ & & $4.20 \pm 2.33$ & & $2.26 \pm 2.79$ \\
\hline & VAS (Rest Pain) & & $1.22 \pm 1.42$ & & $1.78 \pm 1.34$ & & $1.25 \pm 1.14$ & & $2.29 \pm 2.03$ & & $1.55 \pm 1.67$ & & $1.07 \pm 1.67$ \\
\hline \multirow{8}{*}{$\begin{array}{c}\text { MOR-k-1 } \\
\text { [226] }\end{array}$} & $\mathrm{BP}(\%)$ & 5 & $(1-17.5)$ & 1 & $(0.5-3.75)$ & 0.5 & $(0-2)$ & 1 & $(0-3.33)$ & 2 & $(0.5-4.17)$ & 2.5 & $(0.8-4.17)$ \\
\hline & $\mathrm{ZP}(\%)$ & 22.5 & $(12.5-37.5)$ & 16.67 & $7(3.33-30)$ & 15 & $(3.19-20.83)$ & 17.5 & $(4.17-30)$ & 17.5 & $(4.17-31.25)$ & 12.5 & $(2.33-25)$ \\
\hline & $\mathrm{SP}(\%)$ & & $(45-85)$ & 83.33 & $3(60-96.67)$ & 85 & $(73.75-100)$ & 80 & (68.75-100) & 82.5 & $(65-100)$ & 83.33 & $(70-100)$ \\
\hline & FPD Index & 5.21 & $(2.68-7.55)$ & 3.81 & $(2.84-4.69)$ & 2.12 & $(63.64-2.73)$ & 2.12 & (1.43-4.38) & 2.12 & $(1.43-4.35)$ & 4.2 & $(1.75-5.05)$ \\
\hline & $\mathrm{MDC}(\mathrm{ml} / \mathrm{h})$ & 3 & $(1.5-5)$ & 1 & $(0.5-2.5)$ & 1 & $(0.5-1.5)$ & 1 & $(0-2)$ & 1 & $(0.5-2.5)$ & 1 & $(0.1-2)$ \\
\hline & D/D Ratio & 1.05 & $(1-1.35)$ & 1 & $(1-1.04)$ & 1 & $(1-1)$ & 1 & $(1-1.03)$ & 1 & $(1-1.03)$ & 1 & $(1-1.03)$ \\
\hline & VAS (Most Pain) & & $1.69 \pm 2.32$ & & $4.55 \pm 2.29$ & & $0.67 \pm 0.94$ & & $2.33 \pm 3.30$ & & $2.71 \pm 2.63$ & & $1.80 \pm 2.05$ \\
\hline & VAS (Rest Pain) & & $0.95 \pm 1.47$ & & $2.15 \pm 1.42$ & & $0.33 \pm 0.47$ & & $0.43 \pm 0.37$ & & $1.43 \pm 1.13$ & & $1.06 \pm 1.35$ \\
\hline \multirow{8}{*}{$\begin{array}{c}\text { MOR-2 } \\
{[123]}\end{array}$} & $\mathrm{BP}(\%)$ & 12.5 & $(4.17-33.33)$ & 3.3 & $(1-8.33)$ & 4 & $(2.23-8.33)$ & 3.6 & $(2.23-8.33)$ & 3 & $(1-8.33)$ & 2.5 & $(0.83-4.17)$ \\
\hline & $\mathrm{ZP}(\%)$ & 33.33 & $3(20.21-42.5)$ & & $(10-41.67)$ & 16.67 & $(4.12-33.33)$ & 20.83 & $(3.32-36.88)$ & 20.8 & $3(3.32-34.58)$ & 12.5 & $(2.1-29.17)$ \\
\hline & $\mathrm{SP}(\%)$ & 42.5 & $(25-70)$ & 66.67 & $7(47.5-86.88)$ & 79.17 & $(54.17-100)$ & 70.83 & $(50-100)$ & 75 & $(54.38-100)$ & 83.33 & $(62.5-100)$ \\
\hline & FPD Index & 6.26 & $(4.44-9.74)$ & 4.18 & $(3-6.29)$ & 2.21 & $(2.46-5.55)$ & 2.63 & $(1.43-5.88)$ & 2.45 & $(1.43-5.64)$ & 4.21 & $(2.01-5.15)$ \\
\hline & $\mathrm{MDC}(\mathrm{ml} / \mathrm{h})$ & 5 & $(3-8)$ & 2 & $(1-4.15)$ & 2 & $(1-4)$ & 2 & $(1-4)$ & 2 & $(1-3.66)$ & 2 & $(1-3.2)$ \\
\hline & D/D Ratio & 1.13 & $(1.03-1.48)$ & 1 & $(1-1.04)$ & 1 & $(1-1.08)$ & 1 & $(1-1.08)$ & & $(1-1.04)$ & 1 & $(1-1.04)$ \\
\hline & VAS (Most Pain) & & $2.02 \pm 2.86$ & & $3.90 \pm 3.02$ & & $1.75 \pm 2.06$ & & $2.67 \pm 1.75$ & & $4.13 \pm 1.36$ & & $2.49 \pm 2.58$ \\
\hline & VAS (Rest Pain) & & $1.10^{ \pm} 1.49$ & & $2.39 \pm 2.04$ & & $1.28 \pm 1.15$ & & $1.10 \pm 0.89$ & & $1.73 \pm 1.71$ & & $1.64 \pm 1.60$ \\
\hline \multirow{8}{*}{$\begin{array}{c}\text { MOR-k-2 } \\
\text { [75] }\end{array}$} & $\mathrm{BP}(\%)$ & 8.33 & $(2.2-27.08)$ & 2.2 & $(1-4.17)$ & 1 & $(0.33-2.08)$ & 1 & $(0.33-1.5)$ & 2.5 & $(1.2-4.17)$ & 2.5 & $(1-4.17)$ \\
\hline & $\mathrm{ZP}(\%)$ & 29.17 & $7(20.83-37.5)$ & 16.67 & $7(4.17-33.33)$ & 16.67 & $(3.32-29.17)$ & 16.67 & $(2.33-25)$ & 16.6 & $7(2.33-33.33)$ & 16.67 & $(3.86-33.33)$ \\
\hline & $\mathrm{SP}(\%)$ & 58.33 & $3(31.25-73.75)$ & 83.33 & $3(58.33-95.83)$ & 83.33 & $(67.08-100)$ & 83.33 & $(67.08-100)$ & 83.3 & $3(62.5-97.92)$ & 83.33 & $(62.5-100)$ \\
\hline & FPD Index & 5.65 & $(2.8-8.82)$ & 3.83 & $(1.74-5.08)$ & 1.99 & $(2.01-2.77)$ & 1.99 & $(1.43-2.73)$ & 2.21 & $(1.74-5.11)$ & 2.73 & $(1.75-5.15)$ \\
\hline & $\mathrm{MDC}(\mathrm{m} / \mathrm{h})$ & 4 & $(3-7.7)$ & 2 & $(1-3)$ & 1 & $(0.5-2)$ & 1 & $(0.8-2.33)$ & 1.2 & $(1-3)$ & 1.5 & $(1-3.1)$ \\
\hline & D/D Ratio & 1.04 & $(1-1.26)$ & 1 & $(1-1.01)$ & 1 & $(1-1)$ & 1 & $(1-1)$ & 1 & $(1-1)$ & 1 & $(1-1)$ \\
\hline & VAS (Most Pain) & & $1.75 \pm 2.20$ & & $3.72 \pm 1.89$ & & $3.14 \pm 2.47$ & & $3.80 \pm 1.41$ & & $2.87 \pm 2.64$ & & $1.96 \pm 2.2$ \\
\hline & VAS (Rest Pain) & & $0.94 \pm 1.38$ & & $2.04 \pm 1.36$ & & $1.34 \pm 1.34$ & & $1.13 \pm 0.49$ & & $1.20 \pm 0.75$ & & $1.28 \pm 0.93$ \\
\hline
\end{tabular}

Table III Comparison of $P$-value of the FPD index, MDC, D/D ratio, VAS (Most pain), and VAS (Rest pain) at each interval

\begin{tabular}{|c|l|c|c|c|c|c|c|}
\hline \multicolumn{2}{|c|}{} & $0 \sim 4$ & $4 \sim 8$ & $8 \sim 12$ & $12 \sim 16$ & $16 \sim 20$ & $20 \sim 24$ \\
\hline \multirow{3}{*}{ FPD Index } & MOR-1 vs MOR-k-1 & 0.009 & 0.047 & 0.033 & 0.121 & 0.051 & 0.406 \\
\cline { 2 - 8 } & MOR-2 vs MOR-k-2 & 0.38 & 0.027 & 0.037 & 0.015 & 0.473 & 0.614 \\
\hline \multirow{3}{*}{ MDC } & MOR-1 vs MOR-k-1 & 0.334 & 0.101 & 0.036 & 0.044 & 0.074 & 0.415 \\
\cline { 2 - 8 } & MOR-2 vs MOR-k-2 & 0.417 & 0.047 & 0.005 & 0.007 & 0.186 & 0.759 \\
\hline \multirow{2}{*}{ D/D Ratio } & MOR-1 vs MOR-k-1 & 0.834 & 0.985 & 0.092 & 0.234 & 0.677 & 0.111 \\
\cline { 2 - 8 } & MOR-2 vs MOR-k-2 & 0.469 & 0.105 & 0.322 & 0.072 & 0.63 & 0.851 \\
\hline \multirow{2}{*}{$\begin{array}{c}\text { VAS } \\
\text { Most pain) }\end{array}$} & MOR-1 vs MOR-k-1 & 0.139 & 0.329 & 0.006 & 0.019 & 0.782 & 0.975 \\
\cline { 2 - 8 } & MOR-2 vs MOR-k-2 & 0.435 & 0.406 & 0.895 & 0.919 & 0.225 & 0.045 \\
\hline $\begin{array}{c}\text { VAS } \\
\text { (Rest pain) }\end{array}$ & MOR-1 vs MOR-k-1 & 0.487 & 0.146 & 0.044 & 0.684 & 0.048 & 0.380 \\
\cline { 2 - 8 } & MOR-2 vs MOR-k-2 & 0.458 & 0.768 & 0.103 & 0.111 & 0.044 & 0.116 \\
\hline
\end{tabular}

Table IV Pearson product-moment correlation coefficient $r$ value

\begin{tabular}{|l|l|l|l|l|} 
Correlation Coefficient $\boldsymbol{r}$ & MOR-1 & MOR-2 & MOR-K-1 & MOR-K-2 \\
\hline
\end{tabular} \begin{tabular}{|l|l|l|l|l|}
\hline FPD Index VS. VAS (Most pain) & 0.002726 & 0.002596 & 0.0345 & 0.031904 \\
\hline
\end{tabular} \begin{tabular}{|l|l|l|l|l|}
\hline FPD Index VS. VAS (Rest pain) & 0.009828 & 0.012207 & 0.042391 & 0.028997 \\
\hline
\end{tabular} 